\title{
Genome-wide transcriptomic analysis of the response to nitrogen limitation in Streptomyces coelicolor A3(2)
}

Richard A Lewis, Sanjay K Shahi, Emma Laing, Giselda Bucca, Georgios Efthimiou, Michael Bushell and Colin P Smith*

\begin{abstract}
Background: The present study represents a genome-wide transcriptomic analysis of the response of the model streptomycete Streptomyces coelicolor A3(2) M145 to fermentor culture in Modified Evans Media limited, respectively, for nitrogen, phosphate and carbon undertaken as part of the ActinoGEN consortium to provide a publicly available reference microarray dataset.

Findings: A microarray dataset using samples from two replicate cultures for each nutrient limitation was generated. In this report our analysis has focused on the genes which are significantly differentially expressed, as determined by Rank Products Analysis, between samples from matched time points correlated by growth phase for the three pairs of differently limited culture datasets. With a few exceptions, genes are only significantly differentially expressed between the N6/N7 time points and their corresponding time points in the C and P-limited cultures, with the vast majority of the differentially expressed genes being more highly expressed in the N-limited cultures. Our analysis of these genes indicated expression of several members of the GlnR regulon are induced upon nitrogen limitation, as assayed for by $\left[\mathrm{NH}_{4}^{+}\right]$measurements, and we are able to identify several additional genes not present in the GlnR regulon whose expression is induced in response to nitrogen limitation. We also note SCO3327 which encodes a small protein (32 amino acid residues) unusually rich in the basic amino acids lysine (31.25\%) and arginine (25\%) is significantly differentially expressed in the nitrogen limited cultures. Additionally, we investigate the expression of known members of the GlnR regulon and the relationship between gene organization and expression for the SCO2486-SCO2487 and SCO5583-SCO5585 operons.

Conclusions: We provide a list of genes whose expression is differentially expressed in low nitrogen culture conditions, including a putative nitrogen storage protein encoded by SCO3327. Our list includes several genes whose expression patterns are similar to up-regulated members of the GlnR regulon and are induced in response to nitrogen limitation. These genes represent likely targets for future studies into the nitrogen starvation response in Streptomyces coelicolor.
\end{abstract}

\section{Introduction}

It has long been known that the carbon $(\mathrm{C})$, nitrogen $(\mathrm{N})$ and phosphate $(\mathrm{P})$ ratios of Streptomyces media require optimization for secondary metabolite biosynthesis $[1,2]$. As the soil, the natural habitat of the model streptomycete Streptomyces coelicolor, is carbon rich, investigations have focused upon determining the affect

\footnotetext{
* Correspondence: c.p.smith@surrey.ac.uk
Faculty of Heath \& Medical Sciences, University of Surrey, Stag Hill, Guildford,

* Correspondence: c.p.smith@surrey.ac.uk
Faculty of Heath \& Medical Sciences, University of Surrey, Stag Hill, Guildford, Surrey, GU2 7XH, UK
} Surcy, GU2 7XH, $U$ (

on metabolism and gene regulation of limiting the supply of the other two key nutrients, phosphate and nitrogen and Streptomyces nitrogen metabolism has been previously reviewed $[3,4]$. Of the five genes in S. coelicolor which are predicated to encode glutamine synthetase only two $(g \ln A \& g \ln I I)$ [5] are believed to be functional whilst the others $(g \ln A 2, g \ln A 3 \& g \ln A 4)$ are redundant and lack conserved residues involved in catalysis [6]. The housekeeping GSI type enzyme GlnA is expressed at a constant level throughout development [7] and the GSII type enzyme, GlnII, is thought to play a specific 
role in development as it is active only during differentiation of mycelium on solid growth media [8,9]. Following an increase in the $\mathrm{NH}_{4}{ }^{+}$supply GlnA activity is reduced due to it being adenylylated by the adenylyltransferase enzyme, GlnE, and conversely its activity may be restored by de-adenylylation following a decrease in the $\mathrm{NH}_{4}{ }^{+}$supply $[7,10]$. In E. coli GlnE activity is regulated by $G \ln B, G \ln D$ and $G \ln K$ and although homologues of GlnD and GlnK have been identified in $S$. coelicolor their deletion does not affect the regulation of GlnA activity [11]. Under nitrogen limiting conditions GlnD post-translationally modifies the PII protein, GlnK, by adenylylating a conserved tyrosine residue. Furthermore, although this modification is reversible, a secondary modification, the removal of the $\mathrm{N}$-terminal three amino acids of $\mathrm{GlnK}$ is not reversible following a nitrogen starvation shock [11]. Therefore, although it is clear that differences in nitrogen availability result in changes to $\mathrm{GlnD} / \mathrm{K}$ activity, the physiological significance of this phenomenon is unknown. Similarly, the physiological significance of a ncRNA which interferes with the translation of $g \ln A$ mRNA is also unclear [12]. In addition to post-translational modifications genes involved in nitrogen metabolism also seem to be regulated at the level of transcription. The master regulator GlnR [13] has been shown to interact with a consensus sequence present in the upstream regions of $g \ln A$ and the $a m t B-g \ln K-g \ln D$ operon and to up-regulate GlnA activity $[9,14]$. A second potential regulator, GlnRII, was also identified which bound the promoter regions of the $a m t B$ operon, $g \ln A$ and $g \ln I I$, although its functional significance is unclear [9]. More recent studies have extended the Gln $R$ regulon to encompass further genes involved in nitrogen metabolism i.e. the glutamine dehydrogenase gene ( $g d h$; SCO4683), the nitrate reductase gene (nirB; SCO2486) and urease gamma subunit (ureA; SCO1236), in addition to other genes whose involvement in the nitrogen starvation response is unclear [15]. An assimilatory nitrate reductase encoding gene, nas A (SCO2473), whose promoter possesses a variant of the $G \ln R$ binding sequence was also identified [16]. It is likely that the nitrogen and phosphate transcriptional regulatory networks are linked as it has been shown that PhoP represses transcription from the $g \ln R, g \ln A, g \ln I I$ and $a m t B$ promoters, which possess PhoP binding sites [17]. This interaction was first suggested by microarray data [18] and to extend these studies and enhance our understanding of the interactions of different nutrient specific regulatory networks the present study was conceived under the aegis of the European ActinoGEN consortium.

The work presented here was designed to use microarray technology to characterize the differences in transcription which occur in fermentor cultures of the wild- type S. coelicolor A3(2) strain M145 grown in defined, minimal liquid media limited for nitrogen, carbon and phosphate, respectively and provide a publicly available reference dataset. To our knowledge no microarray data relating to Streptomyces coelicolor gene expression under carbon-limited culture conditions have been published. Additionally, the present study represents an advance over earlier microarray studies of gene expression in phosphate limited culture, both in terms of sampling frequency and standardization of culture conditions [18], and also represents an advance over previous studies of gene expression in nitrogen limited culture, both in terms of number of replicates, sampling frequency and gene coverage [15].

\section{Hypothesis}

Growth of Streptomyces coelicolor A3(2) M145 in fermentor cultures limited for nitrogen results in a set of genes being differentially expressed when compared with similar cultures limited for phosphate and carbon respectively.

\section{Materials and methods \\ Strain}

In this study the Streptomyces coelicolor A3(2) strain M145 was used which is a prototrophic derivative of strain A3(2) lacking its two plasmids, SCP1 and SCP2. This is the standard strain used by the ActinoGEN consortium and the strain from which the Streptomyces coelicolor genome sequence was obtained [19].

\section{Media preparation}

Cultures were performed using the following media:Mannitol-Soya (MS) agar [20]. GGI:- Glucose 15 g, Glycerol, $15 \mathrm{~g}$, soya peptone, $15 \mathrm{~g}, \mathrm{NaCl}_{2} 3 \mathrm{~g}, \mathrm{CaCO}_{3} 1 \mathrm{~g}$, $\mathrm{dH}_{2} \mathrm{O}$ to $1 \mathrm{~L}$ followed by adjustment of the $\mathrm{pH}$ to 6.8 and sterilization by autoclaving. GYB:- Glucose $33 \mathrm{~g}$, yeast extract $15 \mathrm{~g}, \mathrm{dH}_{2} \mathrm{O}$ to $1 \mathrm{~L}$ followed by adjustment of the pH to 6.8 and sterilization by filter sterilization. P, N and C- limited Modified Evans Media were prepared as set out in Additional File 1. Glucose solution concentrates (N, P-limited: $700 \mathrm{mM}$, C-limited: $250 \mathrm{mM}$ ) were autoclaved separately from the other mixed media components and added aseptically. Five $\mathrm{ml} / \mathrm{L}$ of Trace Elements $\left(\mathrm{ZnO} 50 \mathrm{mM}, \mathrm{FeCl}_{3} 20 \mathrm{mM}\right.$, $\mathrm{MnCl}_{2} 10 \mathrm{mM}, \mathrm{CuCl}_{2} 10 \mathrm{mM}, \mathrm{CoCl}_{2} 20 \mathrm{mM}, \mathrm{H}_{3} \mathrm{BO}_{3}$ $10 \mathrm{mM}, \mathrm{Na}_{2} \mathrm{MoO}_{4} 0.02 \mathrm{mM}, \mathrm{HCl} 80 \mathrm{ml}, \mathrm{dH}_{2} \mathrm{O}$ up to $1 \mathrm{~L})$ was also added aseptically to the other media components following autoclaving.

\section{Inoculum preparation}

A dense spore suspension was streaked out to produce a confluent lawn on MS agar. Following sporulation a plug was cut with the wide end of a $1 \mathrm{ml}$ pipette tip 
and used to inoculate $50 \mathrm{ml}$ of GGI medium in a $250 \mathrm{ml}$ flask which was incubated for $48 \mathrm{hr}$ at $30^{\circ} \mathrm{C}$ with agitation $(200 \mathrm{rpm})$. Four $\mathrm{ml}$ of this culture was subsequently used to inoculate $50 \mathrm{ml}$ of GYB medium which was incubated for $24 \mathrm{~h}$ at $30^{\circ} \mathrm{C}$ with agitation (200 rpm). The GYB culture was subsequently used to inoculate (10\% final volume) Modified Evans Medium (see Additional File 1) which was incubated for $24 \mathrm{~h}$ at $30^{\circ} \mathrm{C}$ with agitation $(200 \mathrm{rpm})$. This culture was used as the fermentor inoculum $(4 \% \mathrm{v} / \mathrm{v})$. The three step procedure ensured minimal carry-over of rich nutrients from the inoculum production flasks into the fermentor.

\section{Bioreactor culture and sampling}

The inoculum culture $(4 \% \mathrm{v} / \mathrm{v})$ was used to inoculate a $5 \mathrm{~L}$ fermentor vessel (Adaptive Biosystems, Luton, UK) containing $3 \mathrm{~L}$ of Modified Evans Medium. Temperature was maintained at $30^{\circ} \mathrm{C}, \mathrm{pH}$ at 6.8 , agitation $(800 \mathrm{rpm})$ was achieved using a large marine impeller and the aeration rate was $2 \mathrm{~L} / \mathrm{min}$. All cultures were performed in duplicate i.e. the "Inoculum Preparation" and "Bioreactor Culture and Sampling" protocols were repeated for each type of Modified Evans Medium to generate two independent sets of replicate samples per medium type. Samples were taken for biomass determination and RNA isolation at intervals, which were more frequent during the exponential and first transition phases. Three $\times$ five $\mathrm{ml}$ samples were filtered through $0.45 \mu \mathrm{m}$ predried, preweighed nitrocellulose membranes (Millipore, Watford, UK) and the culture filtrate was retained for actinorhodin assay [21] and nutrient analyses $\left(\mathrm{PO}_{4}^{-}\right.$, $\mathrm{NH}_{4}{ }^{+}$and Glucose) using a Merck RQFlex $10^{\mathrm{TM}}$ reflectometer. The nitrocellulose membranes were rinsed three times with ultrapure water, microwaved twice at $650 \mathrm{~W}$ for five min and allowed to cool in a dessicator before the weight was determined. For RNA isolation culture samples were added to two volumes of RNAprotect (Qiagen) in a Falcon tube and vortex-mixed. The mixture was incubated for $10 \mathrm{~min}$ at room temperature before centrifugation at $3500 \mathrm{rpm}$ for $10 \mathrm{~min}$. The supernatant was decanted and any excess liquid removed by inverting the tube before storage of the cell pellet at $-80^{\circ} \mathrm{C}$. For a limited number of time points cell pellets derived from $5 \mathrm{ml}$ of culture were harvested for undecylprodiginine assay [22].

\section{Nucleic acid isolation and microarray techniques}

Protocols for RNA isolation using the "Total RNA extraction by Tissue Lyzer" are provided at our microarray resource website [23]. The quality of the RNA obtained was assessed by running a Bioanalyzer Prokaryote Total RNA Nano chip (Agilent) and only RNA having a RNA Integrity Number of 6.5 or above was used in subsequent work. Protocols for S. coelicolor M145 genomic DNA isolation and labeling (Cy5), together with the labeling of cDNA (Cy3) are as described at our microarray resource website [23] and hybridizations were set up using our own in-house printed Streptomyces coelicolor oligonucleotide based spotted microarrays [23], as described at the microarray resource website [23].

\section{Microarray data processing and analysis}

The microarrays were scanned using an Agilent scanner (5 $\mu \mathrm{m}$ resolution).

Images were imported into the software BlueFuse (Version 3.1; BlueGnome Ltd, Cambridge) for feature extraction and spot quantification as detailed in [24]. BlueFuse output files were imported into the statistical computing environment $\mathrm{R}$ (Version 2.5.1) [25,26] for data normalization using the Limma package $[27,28]$. Within-array global median normalization of $\log _{2}$ cDNA/gDNA ratios was applied to each array in the analysis, ignoring control and flagged spots. Subsequent $\log _{2}$ ratios were scaled to have the same medianabsolute-deviation (MAD) across all arrays $[27,28]$. Flagging was applied to the normalized data such that probes with a PON CH2 (probability of a good signal in the genomic DNA channel of the array, as designated by BlueFuse) value less than 0.5 were assigned 'NA'. Genes that did not have at least one good probe for each condition/replicate were filtered out and not included in any downstream analysis. Due to the high stringency of this filtering only 5,060 genes, which gave a signal in all of the 38 samples used in the study, were analyzed using Rank products analysis [29]. The R package RankProd [30] was used to perform Rank products analysis with default parameters for each pairwise condition/ time-point comparison in the dataset. Following this, lists of significantly (Rank Products pfp value of $\leq 0.15$ ) differentially expressed genes were identified for each pair of time points. In this report our analysis has focused on the genes which are significantly differentially expressed between samples from matched time points (T1-T7) for the three pairs of datasets i.e. $\mathrm{N}$ vs $\mathrm{C}, \mathrm{N}$ vs $\mathrm{P}$, and $\mathrm{C}$ vs $\mathrm{P}$ as set out in Additional File 2. Alignment of the growth curves (Figure 1) ensured the matched time points from differently limited cultures were correlated by growth phase. Although seven time points from the $\mathrm{C}$ and $\mathrm{N}$-limited cultures could be matched to one another, due to the lower sampling frequency for the P-limited cultures only five time points could be matched with corresponding pairs of time points from the $\mathrm{N}$ and C-limited cultures. This approach was adopted so as to nullify the effect of interference from growth phase-dependent gene expression on the results and ensure that the lists of significantly differentially expressed genes generated are directly 


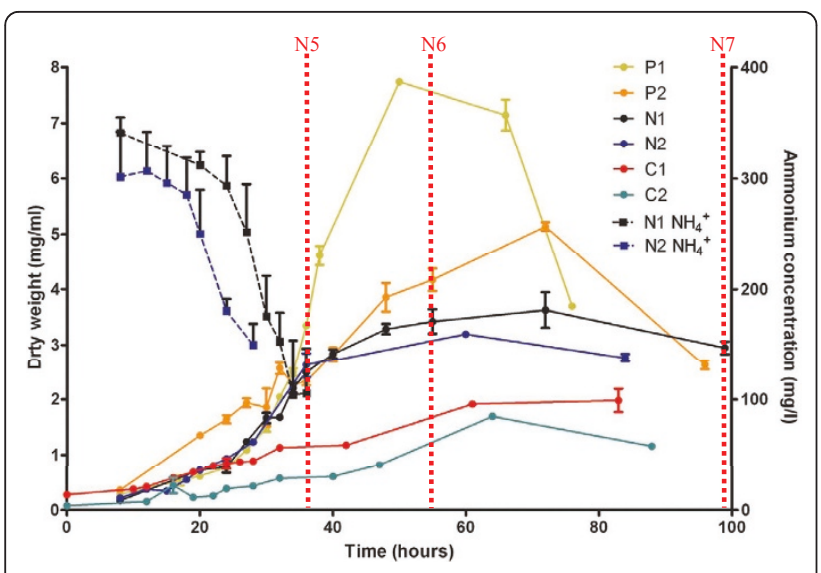

Figure 1 Growth curves for duplicate fermentor cultures of $S$. coelicolor A2(3) M145 grown in N, P and C-limited Modified Evans Medium. Three dry weights per sample per time point were averaged to provide each datum. To align the exponential phases of the cultures with the $\mathrm{N} 1$ culture the time points for the $\mathrm{N} 2, \mathrm{C} 1$ and C2 cultures have been shifted by $-12,-8$ and $-8 \mathrm{~h}$ respectively. The $\left[\mathrm{NH}_{4}{ }^{+}\right]$assay data (where available) for the N-limited cultures are also shown. The N5-7 time points, aligned to the N1 growth curve, are indicated by dashed red bars.

attributable to differences in nutrient limitation. The microarray data has been deposited with ArrayExpress (Accession number: E-MAXD-59).

\section{Results and Discussion}

The growth curves of the cultures grown in N, P and C-limited media are strikingly different, (Figure 1) as are the amounts of actinorhodin and undecylprodiginine produced (see Additional Files 3, 4 \&5). The C-limited cultures produced less biomass and pigmented antibiotics than the $\mathrm{N}$ and $\mathrm{P}$-limited cultures whereas the $\mathrm{P}$-limited cultures gave the highest biomass readings and production of actinorhodin and undecylprodiginine. It is clear from the metabolite measurements that the $\mathrm{NH}_{4}{ }^{+}, \mathrm{PO}_{4}{ }^{-}$, and glucose levels in the N, P, C-limited cultures decrease during the culture period to low, and presumably, limiting levels (see Additional File 6).

Examination of the gene lists obtained from Rank Products analysis indicate that no genes are significantly differentially expressed between the $\mathrm{C}$ and P-limited cultures. Moreover, with a few exceptions, genes are only significantly differentially expressed between the N6 and N7 time points and their corresponding time points in the $\mathrm{C}$ and P-limited cultures, with the vast majority of the genes being more highly expressed in the $\mathrm{N}$ limited cultures. Most of the significantly differentially expressed genes were identified as such at both the N6 and the N7 time points in both the $\mathrm{N}$ vs $\mathrm{C}$ and the $\mathrm{N}$ vs $\mathrm{P}$ comparisons. It appears, prima facie, that either our cultures were more successfully limited with respect to nitrogen than they were with respect to carbon or phosphate, or that genes which are differentially expressed in $\mathrm{P}$ and $\mathrm{C}$-limited media are not represented in our dataset, or that nitrogen limitation has a greater effect on differential gene expression than either carbon or phosphate limitation. Our analysis of the present dataset, focusing on the genes which are significantly differentially expressed between the N6 and $\mathrm{N} 7$ time points and their corresponding time points in the $\mathrm{C}$ and P-limited cultures (see Additional File 7), was conducted according to the last premise.

The results indicated that the majority of genes may be categorized into three main gene Expression Categories (I, II or III) as follows. Firstly, (I) genes which have fairly constant, elevated expression in the $\mathrm{N}$-limited cultures relative to the P-limited cultures, and whose expression is also low, or decreases with time across the C-limited cultures. Secondly (II) genes whose expression increases relatively uniformly across the $\mathrm{N}$-limited culture time points to an elevated level relative to the $\mathrm{C}$, and P- limited cultures. Thirdly, (III) and most interestingly, genes whose expression is elevated in the N6 and/ or N7 time points relative to both the early N-limited culture time points and the $\mathrm{C}$ and P-limited cultures.

Regarding the genes of Expression Category (I), clearly, the genes have been identified through their high expression under nitrogen limiting conditions relative to the other limited cultures (Figure 2). However their expression patterns do not resemble those of the GlnR regulon up-regulated genes (see below) and their annotations do not suggest involvement in nitrogen metabolism. For example, the regulatory WblA-encoding gene, SCO3579, which is a pleiotropic down-regulator of antibiotic biosynthesis [31,32] is present in this category. However, we note that SCO3327 (Figure 2) encodes a small (32 amino acid) protein which comprises an unusually high proportion of basic amino acid residues $(>50 \%)$ with long chain $\mathrm{R}$ groups containing nitrogen i.e. arginine $(25 \%)$ and lysine (31.25\%). Two proteins rich in arginine have previously been identified whose genes have promoters which comprise putative GlnR binding sites i.e. SCO1863 is a 406 amino acid protein (see Figure 3) comprising 83 arginine residues (20.4\%) [9], and SCO2195 is a 71 amino acid protein comprising 13 arginines (18.3\%) [15]. Although all three proteins lack direct sequence similarity with one another they are similar in terms of having a high percentage of arginine residues, which is considerably higher than the standard S. coelicolor protein arginine composition of 8\% [20]. The role/s of these proteins in nitrogen limited metabolism are unclear, although it has been suggested that SCO2195 is involved in RNA binding [15] and that SCO1863 may be a nitrogen storage protein [3] reflecting the fact that arginine is the most nitrogen rich amino acid comprising three atoms of nitrogen per residue. 


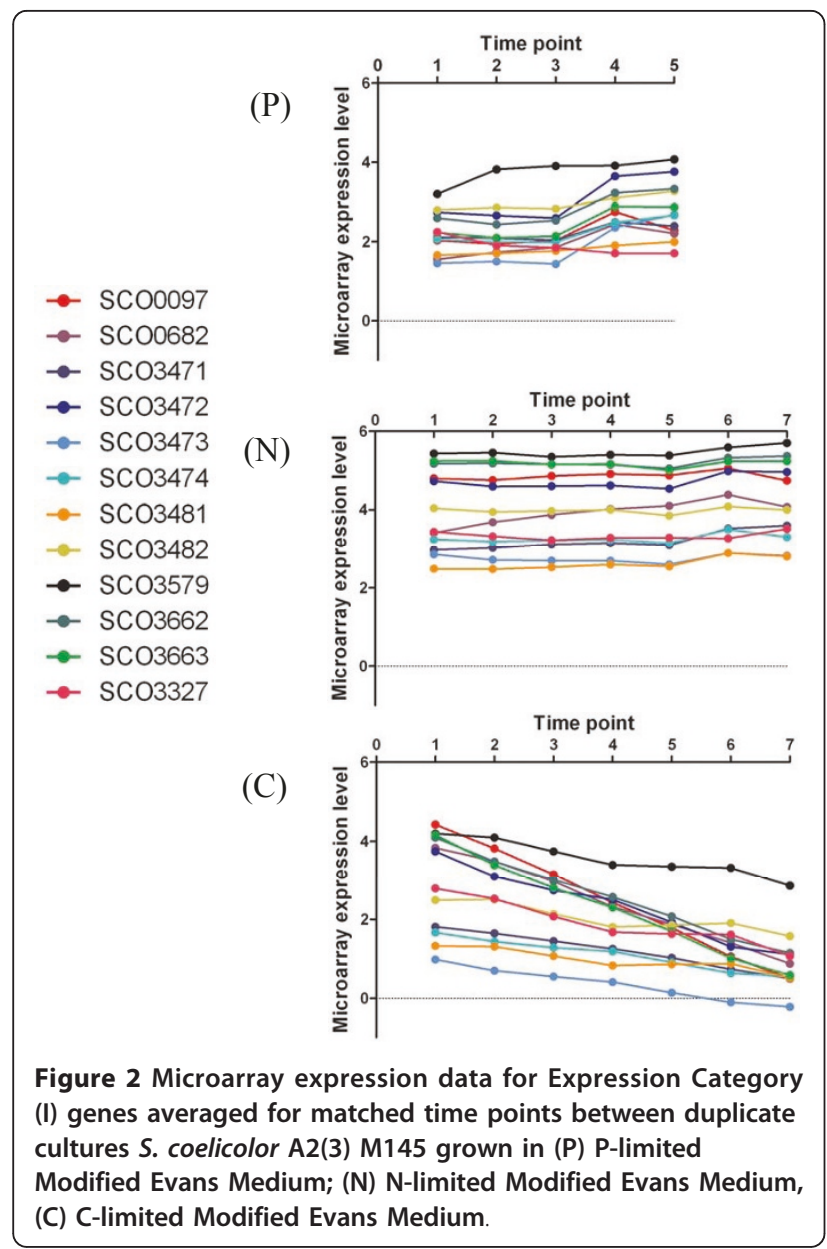

It is clear that many of the genes comprising Expression Category (I) are involved in carbohydrate metabolism. For example, they include an operon comprising dagA (SCO3471) which encodes an extracellular agarase, a putative aldolase (SCO3473), a sugar kinase (SCO3474) together with the co-expressed SCO3472 (a transposase remnant). A glycosyltransferase homologue SCO3481 and a sugar permease (SCO3482) are also presumably expressed as an operon. The down regulation of these carbohydrate metabolism genes in the carbon limited culture may be a response to lack of substrate, however, we speculate that the differential expression of these genes under different nutrient limitations is due to a complex network of regulatory interactions which we are only now beginning to explore. For example, it is known that there are links between nitrogen and carbon metabolism, for example, mediated by dasR [33] and the links between GlnR and PhoP regulatory networks are mentioned above $[17,18]$.

The second Expression Category of genes (II) comprises several regulatory genes, for example, the anti-sigma factor, SCO4677 which is involved in differentiation [34] and

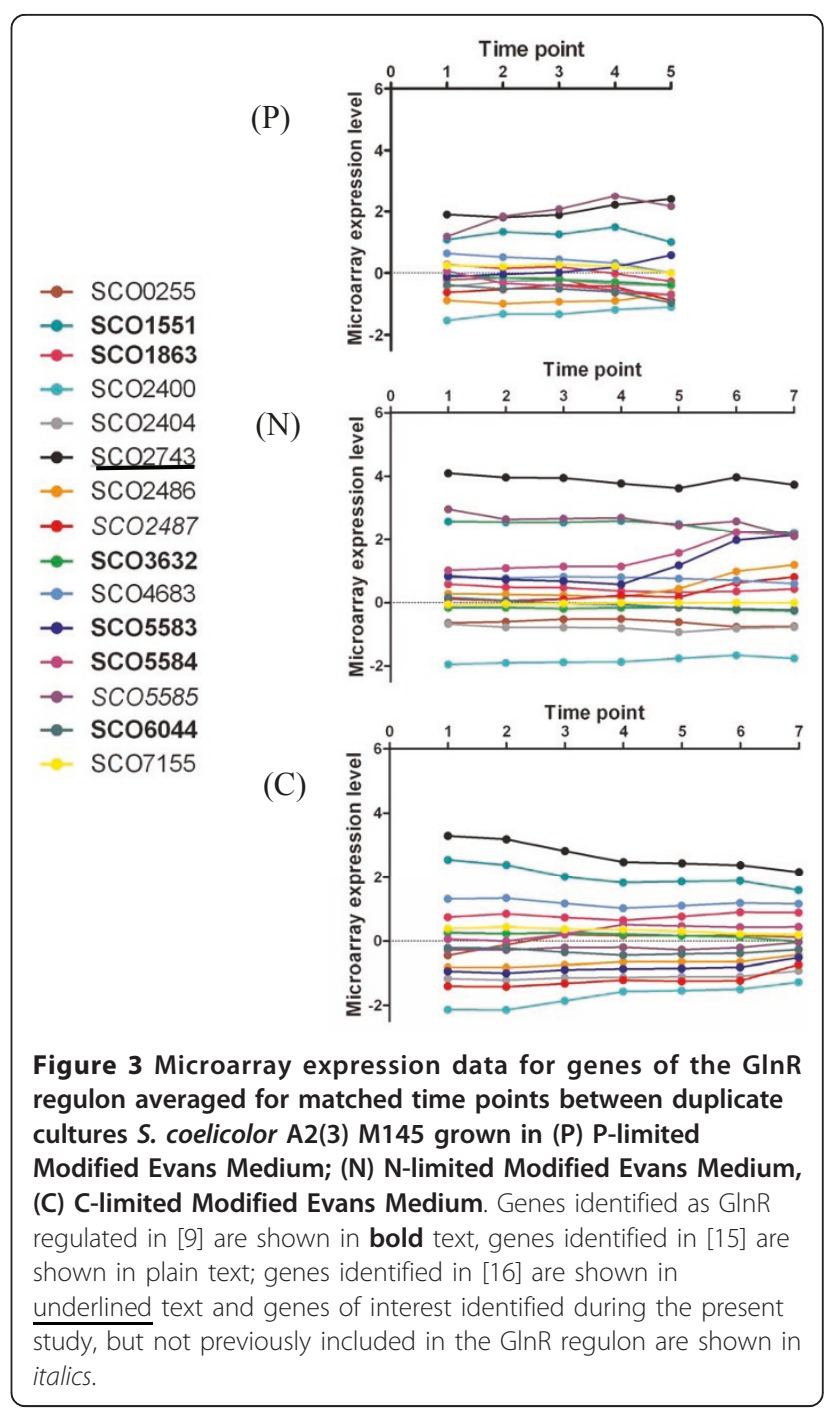

the sigma factor-like SCO3323 (see Figure 4). Genes involved in antibiotic biosynthesis/regulation are also represented and include the actinorhodin transporter SCO5083 (data not shown) and the transcriptional regulator afsR2 (SCO4425).

Several of the significantly differentially expressed genes of the third Expression Category (III) are known to be members of the GlnR regulon and are involved in nitrogen metabolism (see Figure 3). These include the nitrite reductase gene, nirB (SCO2486) together with the ammonium transporter protein amtB (SCO5583) and the gene encoding the PII protein $\operatorname{gln} K$ (SCO5584). Examination of their expression profiles indicates that their expression increases between time points N5 and N6 and is not a transient phenomenon as their expression remains elevated into N7. This phenomenon may be explained by consideration of the $\mathrm{NH}_{4}{ }^{+}$concentration data (Figure 1) which indicates that the ammonium ion concentration falls steeply in the nitrogen limited 


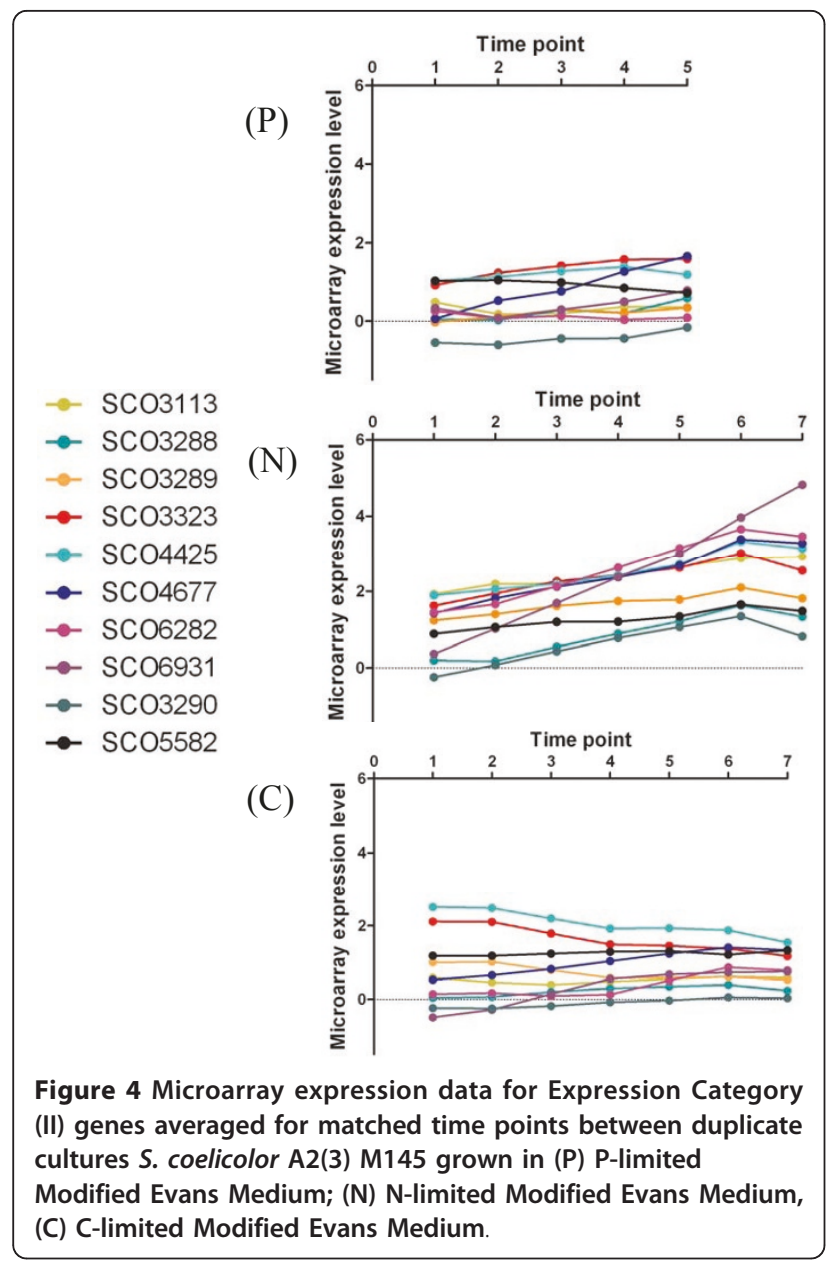

cultures during exponential phase to concentrations below the detection limits of the assay. Presumably this decrease in available nitrogen leads to the culture becoming nitrogen limited during the T5-T6 interval, so triggering the up-regulation of genes involved in nitrogen uptake and metabolism.

Although they are not significantly differentially expressed other genes of the GlnR regulon which are present in our dataset include the NADP-specific glutamate dehydrogenase gene $g d h$ (SCO4683), SCO0255, SCO2400, SCO2404, SCO1863, SCO7155 and the assimilatory nitrate reductase gene nasR (SCO2473) [16]. Their expression profiles together with the other members of the GlnR regulon represented in the dataset are presented in Figure 3 (genes identified as GlnRregulated in [9] are shown in bold text, genes identified in [15] are shown in plain text; genes identified in [16] are shown in underlined text). Given the different culture conditions used and gene expression determination methodologies employed it is difficult to compare our expression data with previous results relating to the transcription of these genes $[9,15,16]$. However, we note that our results regarding up-regulation of SCO2486 and SCO5583/5584 in nitrogen limiting conditions are consistent with previous data $[9,15]$. Additionally, our data indicating the non-alteration of expression of SCO7155 under nitrogen limitation conditions is consistent with previous results [15] and our results which show SCO0255, SCO2400 and SCO2404 are being expressed at a constant, low level are also consistent with the previously reported repression of these genes under nitrogen limiting conditions [15].

We examined the expression profiles of the other significantly differentially expressed genes to identify other genes whose expression patterns are similar to those of SCO2486, SCO5583 and SCO5584 and whose expression may be induced as a result of nitrogen limitation. The expression profiles of these genes are shown in Figure 5. Furthermore, we have also identified genes which are significantly differentially expressed between the N1 time point and successive N-limited culture time points. The majority of these were already represented in the gene lists of significantly differentially expressed

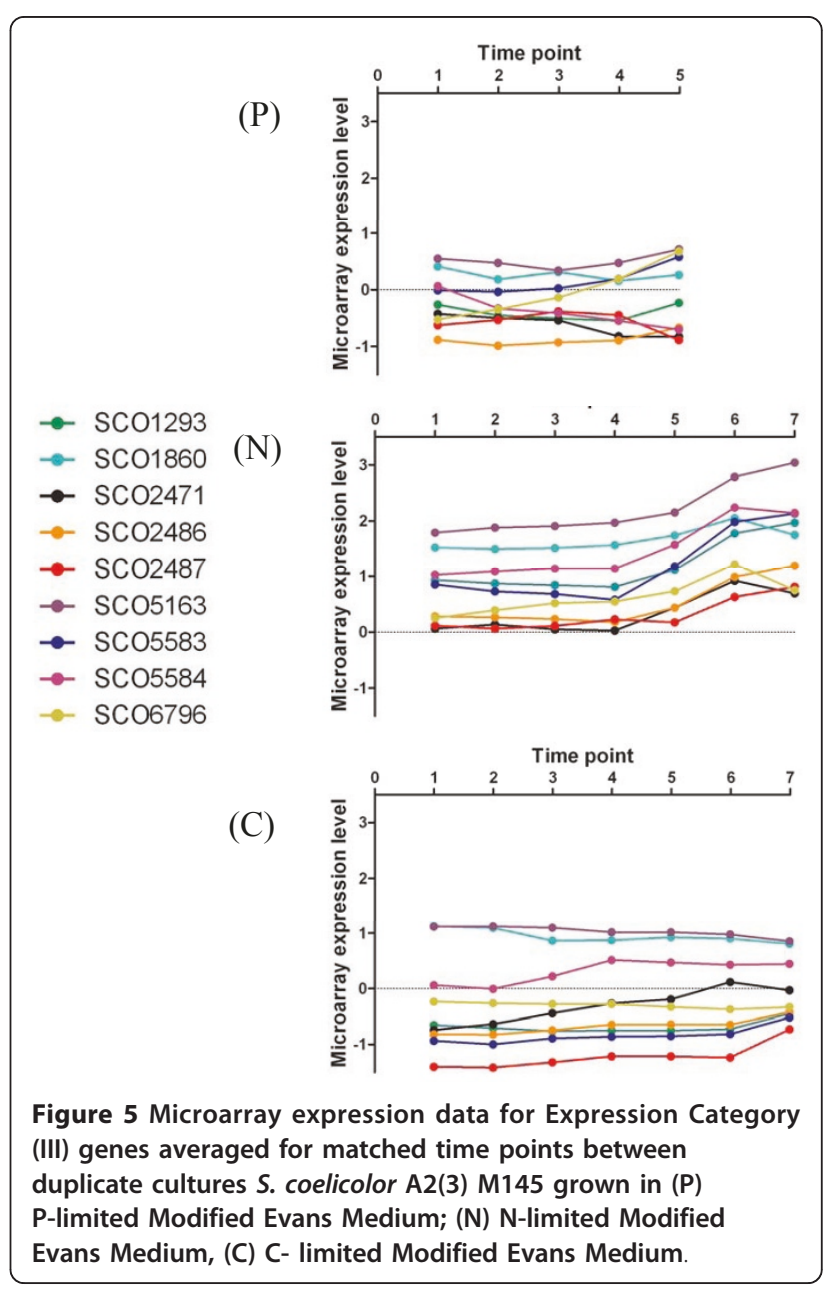


genes identified as a result of comparing the $\mathrm{N}$ limited cultures with those of the $\mathrm{C}$ and $\mathrm{P}$ limited cultures (see Additional File 7). However, an additional five genes were identified (see Additional File 7) whose expression patterns are consistent with them being members of Expression Category II. These include SCO3290 (Figure 4) whose expression profile correlates with those of SCO3288 \& SCO3289 (Figure 4) which are present in our list of differentially regulated genes. We cannot however exclude the possibility that the expression of these five genes is growth phase dependent and is induced on entry into stationary phase. For example, although SCO5582 expression is up-regulated (Figure 4) it seems to encode a regulator with sequence similarity to a sporulation associated protein.

We have analyzed the upstream regions (up to $750 \mathrm{bp}$ ) of all of the significantly differentially expressed genes identified in the present study for potential transcription factor/repressor binding motifs using the sequence motif algorithims MEME [35] and GLAM2, [36] in addition to manual inspection of the sequences. Barring the GlnR binding sites known to exist upstream of members of the GlnR regulon we have been unable to identify further potential protein binding target sequences, excepting two potential "a-site" motifs (GTAAC \& GTAAT) separated by $17 \mathrm{nt}$ upstream of SCO5163 (-141 to -168) which may represent a nas $A$ type GlnR-binding element [16].

We have also used the STRING 8.2 database of protein-protein interactions [37] to search for known interactions between the genes. We note that SCO1861 is linked to SCO5583 and SCO5584 and that SCO1860 is present in our list of significantly differentially expressed genes whilst SCO1863 (the first gene in the SCO18631860 operon) has been identified as possessing a putative upstream GlnR binding site [9]. Barring other obvious associations i.e. consecutive genes present in operons or well known groupings i.e. the GlnR regulon we have been unable to identify further interactions between the significantly differentially expressed genes products.

The gene annotations of the majority of the significantly differentially expressed genes are insufficiently detailed to allow their assignment to specific roles in nitrogen limited metabolism. However, we note that SCO1293 whose expression appears to be induced following nitrogen limitation (Figure $5 \mathrm{~N}$ ) encodes a product with sequence similarity to $\mathrm{N}$-acetylglutamate synthetases. Interestingly also we find that the nitrate reductase large subunit gene, nirB, (SCO2487) is significantly differentially expressed and that its expression profile closely matches that of the upstream nitrite reductase gene $\operatorname{nir} B$ (Figure $5 \mathrm{~N}$ ) which is known to be regulated by GlnR. This result is consistent with the arrangement of the genes in the chromosome as the
SCO2487 coding sequence overlaps SCO2486, and the two genes are presumably co-expressed in an operon. We therefore propose the inclusion of SCO2487 in the GlnR regulon.

Similarly, we also note that the expression profiles of SCO5583 and SCO5584 (Figure 5N) closely match one another which are consistent with previously reported (RT)-PCR results [9]. Our data are also consistent with genetic organization i.e. the sequences of SCO5583 and SCO5584 overlap and are co-transcribed in a single mRNA [9]. Although the gene immediately downstream, i.e. $\operatorname{gln} D$ (SCO5585) which encodes an adenylyltransferase responsible for regulating GlnK activity via post translational modification is present in our list of significantly differentially expressed genes it appears from our data that its expression profile differs from those of SCO5583 and SCO5584. Interestingly, although it has been shown that SCO5584 and SCO5585 are transcriptionally linked the presence of an additional SCO5585 promoter was not ruled out [9] and our results tend to suggest that the presence of an additional promoter is indeed likely explaining the differential regulation of SCO5585 with respect to SCO5583 \& SCO5584 (Figure $3 \mathrm{~N}$ ).

\section{Conclusions}

The present study represents an investigation designed to identify genes of interest involved in Streptomyces nitrogen metabolism which could not be identified simply by sequence similarity searches. In this aim we were successful and we identify a number of genes as targets for future investigation. Of particular interest are the putative nitrogen storage protein encoded by SCO3327, and the up-regulated genes SCO1293 and SCO5163 which may play roles in the nitrogen starvation response. We expect that the present study will serve to inform and stimulate future research into the genes identified herein.

\section{Additional material}

Additional File 1: Table providing details of the Modified Evans Media ( $\mathrm{P}, \mathrm{C}$ and $\mathrm{N}$-limited) used in the present study.

Additional File 2: Table providing details of matched pairs of samples from duplicate cultures S. coelicolor A2(3) M145 grown in differently limited (N, P, C) Modified Evans Medium and correlated between the differently limited cultures according to time point i.e. growth phase.

Additional File 3: Results of actinorhodin assays of samples from S. coelicolor A2(3) M145 cultures grown in differently limited (N, P, C) Modified Evans Medium. Data are averaged results from two technical replicates.

Additional File 4: Results of undecylprodiginine assays of samples from S. coelicolor A2(3) M145 cultures grown in differently limited (N, P, C) Modified Evans Medium. Data are averaged results from two technical replicates. 
Additional File 5: Graph of results of actinorhodin and undecylprodiginine assays of samples from S. coelicolor A2(3) M145 cultures grown in differently limited (N, P, C) Modified Evans Medium.

Additional File 6: Results of phosphate, ammonium and glucose assays of samples from S. coelicolor A2(3) M145 cultures grown in differently limited (N, P, C) Modified Evans Medium. "ND" indicates the concentration was below the detection threshold of the assay system. Data are averaged results from at least two technical replicates with the exception of the P2 phosphate assay which represents the results of a single replicate.

Additional File 7: List of genes significantly differentially expressed at the N6/N7 time points relative to comparison time points. Details of their functional annotations and Expression Category (I, II or III) as defined in the "Results \& Discussion" are provided. Those genes identified as a result of comparing the C-limited time points with the N-limited time points are marked ${ }^{*}$, those identified as a result of comparing the P-limited time points with the N-limited time points are marked \# and those identified as a result of comparing the $\mathrm{N} 1$ nitrogen time point with subsequent $\mathrm{N}$-limited time points are marked +. Unmarked genes were identified as a result of comparisons between the $\mathrm{N}$ - limited time points and both the $\mathrm{C}$ and $\mathrm{P}$-limited time points.

\section{Acknowledgements}

We thank Mervyn Bibb for providing the original S. coelicolor M145 strain. We thank Noel Wardell for helpful technical discussions on running fermentors. This work was funded by the European Commission (FP6 Programme ActinoGEN IP005224 to C.P.S.).

\section{Authors' contributions}

RAL did the microarray experiments, metabolite assays, microarray data analysis and wrote the manuscript. EL processed the microarray data and contributed to the manuscript. SKS did the fermentor cultures. GB assisted with the microarray experiments and microarray data analysis. GE did the pigmented antibiotic assays. MB assisted with the fermentor experiments. CPS designed the study and directed the work. All of the authors have read and approved the final manuscript.

\section{Competing interests}

The authors declare that they have no competing interests.

Received: 5 November 2010 Accepted: 23 March 2011

Published: 23 March 2011

\section{References}

1. Hobbs G, Frazer CM, Gardner DCJ, Flett F, Oliver SG: Pigmented antibiotic production by Streptomyces coelicolor A3(2): kinetics and influence of nutrients. Journal of General Microbiology 1990, 136:2291-2296.

2. Fryday A: The application of materials balancing to the characterization of sequential secondary metabolite formation in Streptomyces cattleya NRRL 8057. Journal of General Microbiology 1983, 129:1733-1741.

3. Reuther J, Wohlleben W: Nitrogen metabolism in Streptomyces coelicolor: Transcriptional and post-translational regulation. Journal of Molecular Microbiology and Biotechnology 2007, 12:139-146.

4. Harper C, Hayward D, Wiid I, van Helden P: Regulation of nitrogen metabolism in Mycobacterium tuberculosis: a comparison with mechanisms in Cornebacterium glutamicum and Streptomyces coelicolor. IUMB Life 2008, 60:643-650.

5. Wray LV, Fisher SH: Cloning and nucleotide sequence of the Streptomyces coelicolor gene encoding glutamine synthetase. Gene 1988, 71:247-256.

6. Rexer HU, Schaberle T, Wohlleben W, Engels A: Investigation of the functional properties and regulation of three glutamine synthetaselike genes in Streptomyces coelicolor A3(2). Arch. Microbiol 2006, 186:447-458.

7. Fisher SH, Wray LV: Regulation of glutamine synthetase in Streptomyces coelicolor. Journal of Bacteriology 1989, 171:2378-2383.
8. Weisschuh N, Fink D, Vierling S, Bibb MJ, Wohlleben W, Engels A: Transcriptional analysis of the gene for glutamine synthetase II and two upstream genes in Streptomyces coelicolor A3(2). Molecular \& General Genetics 2000, 264:461-469.

9. Fink D, Weisschuh N, Reuther J, Wohlleben W, Engels A: Two transcriptional regulators $\mathrm{G} \ln R$ and $\mathrm{G} \ln R \mathrm{Il}$ are involved in regulation of nitrogen metabolism in Streptomyces coelicolor A3(2). Molecular Microbiology 2002, 2:331-347.

10. Fink D, Falke D, Wohlleben W, Engels A: Nitrogen metabolism in Streptomyces coelicolor A3(2): modification of glutamine synthetase by an adenylyltransferase. Microbiology 1999, 145:2315-2322.

11. Hesketh A, Fink D, Gust B, Rexer HU, Scheel B, Chater K, Wohlleben W, Engels A: The GInD and GlnK homologues of Streptomyces coelicolor A3 (2) are functionally dissimilar to their nitrogen regulatory system counterparts from enteric bacteria. Molecular Microbiology 2002, 2:319-330.

12. D'Alia D, Nieselt K, Steigle S, Muller J, Verberg I, Takano E: Noncoding RNA of Glutamine synthetase I modulates antibiotic production in Streptomyces coelicolor A3(2). Journal of Bacteriology 2002, 192:1160-1164.

13. Wray LV, Fisher SH: The Streptomyces coelicolor glnR gene encodes a protein similar to other bacterial response regulators. Gene 1993, 130:145-150.

14. Wray LV, Atkinson MR, Fisher SH: Identification and cloning of the $g \ln R$ locus, which is required for transcription of the $g \ln A$ gene in Streptomyces coelicolor A3(2). Journal of Bacteriology 1991, 173:7351-7360

15. Tiffert $Y$, Supra P, Wurm R, Wohlleben W, Wagner R, Reuther J: The Streptomyces coelicolor GlnR regulon: identification of new GlnR targets and evidence for a central role of GlnR in nitrogen metabolism in actinomycetes. Molecular Microbiology 2008, 67:861-880.

16. Wang J, Zhao G-P: GlnR positively regulates nas $A$ transcription in Streptomyces coelicolor. Biochemical and Biophysical Research Communications 2009, 386:77-81.

17. Rodriguez-Garcia A, Sola-Landa A, Apel K, Santos-Beneit F, Martin JF: Phosphate control over nitrogen metabolism in Streptomyces coelicolor: direct and indirect negative control of $g \ln R, g \ln A, g \ln l \mid$ and $a m t B$ expression by the response regulator PhoP. Nucleic Acids Research 2009, 37:3230-3242.

18. Rodriguez-Garcia A, Barreiro C, Santos-Beneit F, Sola-Landa A, Martin JF: Genome wide transcriptomic and proteomic analysis of the primary response to phosphate limitation in Streptomyces coelicolor M145 and in a DeltaphoP mutant. Proteomics 2007, 14:2410-2429.

19. Bentley SD, Chater KF, Cerdeno-Tarraga AM, Challis GL, Thomson NR, James KD, Harris DE, Quail MA, Kieser H, Harper D, Bateman A, Brown S, Chandra G, Chen CW, Collins M, Cronin A, Fraser A, Goble A, Hidalgo J, Hornsby T, Howarth S, Huang CH, Kieser T, Larke L, Murphy L, Oliver K, O'Neil S, Rabbinowitsch E, Rajandream MA, Rutherford K, Rutter S, Seeger K, Saunders D, Sharp S, Squares R, Squares S, Taylor K, Warren T, Wietzorrek A, Woodward J, Barrell BG, Parkhill J, Hopwood DA: Complete genome sequence of the model actinomycete Streptomyces coelicolor A3(2). Nature 2002, 417:141-147.

20. Kieser T, Bibb MJ, Buttner MJ, Chater KF, Hopwood DA: Practical Streptomyces Genetics Norwich, UK: The John Innes Foundation; 2000.

21. Bystrykh LV, Fernandez-Moreno MA, Herrema JK, Malpartida F, Hopwood DA, Dijkhuizen L: Production of actinorhodin-related "blue pigments" by Streptomyces coelicolor A3(2). Journal of Bacteriology 1996, 178:2238-2244.

22. Tsao SW, Rudd BA, He XG, Chang CJ, Floss HG: Identification of a red pigment from Streptomyces coelicolor A3(2) as a mixture of prodigiosin derivatives. Journal of Antibiotics (Tokyo) 1985, 38:128-131.

23. StreptoBASE - 'omics data for Streptomyces (at the University of Surrey). [http://unis.streptobase.org/].

24. Hesketh A, Bucca G, Laing E, Flett F, Hotchkiss G, Smith CP, Chater KF: New pleiotropic effects of eliminating a rare tRNA from Streptomyces coelicolor revealed by combined proteomic and transcriptomic analysis of liquid cultures. BMC Genomics 2007, 8:261.

25. R Development Core Team. [http://www.R-project.org].

26. $R$ : A language and environment for statistical computing $R$ Foundation for Statistical Computing: Vienna, Austria; 2005, ISBN 3-900051-07-0.

27. Smyth GK: Limma: linear models for microarray data. In Bioinformatics and Computational Biology Solutions using $R$ and Bioconductor. Edited by: 
Gentleman R, Carey V, Dudoit S, Irizarry R, Huber W. New York: Springer; 2005:397-420.

28. Smyth GK, Speed TP: Normalization of CDNA microarray data. Methods 2003, 31:265-273.

29. Breitling R, Armengaud P, Amtmann A, Herzyk P: Rank products: a simple, yet powerful, new method to detect differentially regulated genes in replicated microarray experiments. FEBS Letters 2004, 573:83-92.

30. Hong F, Breitling R, McEntee CW, Wittner BS, Nemhauser JL, Chory J: RankProd: A Bioconductor package for detecting differentially expressed gene in meta-analysis. Bioinformatics 2006, 22:2825-2827.

31. Yang Y-H, Kim J-N, Song E, Kim E, Oh M-K, Kim B-G: Finding new pathway specific regulators by clustering method using threshold standard deviation based on DNA chip data of Streptomyces coelicolor. Applied and Environmental Microbiology 2008, 80:709-717.

32. Kang SH, Huang J, Lee H-N, Hur Y-A, Cohen SN, Kim E-S: Interspecies DNA microarray analysis identifies WbIA as a pleiotropic down-regulator of antibiotic biosynthesis in Streptomyces. Journal of Bacteriology 2007, 189:4315-4319.

33. Rigali S, Nohaft H, Noens EE, Schlicht M, Colson S, Muller M, Joris B, Koerten HK, Hopwood DA, Titgemeyer F, van Wezel GP: The sugar phosphotransferase system of Streptomyces coelicolor is regulated by the GntR-family regulator DasR and links N-acetylglucosamine metabolism to the control of development. Molecular Microbiology 2006, 61:1237-1251.

34. Kim ES, Song JY, Kim DW, Chater KF, Lee KJ: A possible extended family of regulators of sigma factor activity in Streptomyces coelicolor. Journal of Bacteriology 2008, 190:7559-7566.

35. Bailey $T L$, Elkan C: Fitting a mixture model by expectation maximization to discover motifs in biopolymers. Proceedings of the Second International Conference on Intelligent Systems for Molecular Biology Menlo Park, California. AAAI Press; 1994, 28-36.

36. Frith MC, Saunders NFW, Kobe B, Bailey TL: Discovering sequence motifs with arbitrary insertions and deletions. PLoS Computational Biology 2008, 4:e1000071.

37. String- functional protein association networks. [http://string-db.org/].

doi:10.1186/1756-0500-4-78

Cite this article as: Lewis et al: Genome-wide transcriptomic analysis of the response to nitrogen limitation in Streptomyces coelicolor A3(2). BMC Research Notes 2011 4:78.

\section{Submit your next manuscript to BioMed Central and take full advantage of:}

- Convenient online submission

- Thorough peer review

- No space constraints or color figure charges

- Immediate publication on acceptance

- Inclusion in PubMed, CAS, Scopus and Google Scholar

- Research which is freely available for redistribution

Submit your manuscript at www.biomedcentral.com/submit 